\title{
Cryptococcus neoformans Escape From Dictyostelium Amoeba by Both WASH-Mediated Constitutive Exocytosis and Vomocytosis
}

\author{
Rhys A. Watkins ${ }^{1,2 \dagger}$, Alexandre Andrews ${ }^{1,2+}$, Charlotte Wynn ${ }^{1,2}$, Caroline Barisch ${ }^{3}$, \\ Jason S. King ${ }^{1,4 *}$ and Simon A. Johnston ${ }^{1,2 *}$
}

${ }^{1}$ Bateson Centre, University of Sheffield, Sheffield, United Kingdom, ${ }^{2}$ Department of Infection Immunity and Cardiovascular Disease, Medical School, University of Sheffield, Sheffield, United Kingdom, ${ }^{3}$ Department of Biochemistry, Faculty of Science, University of Geneva, Geneva, Switzerland, ${ }^{4}$ Department of Biomedical Sciences, University of Sheffield, Sheffield, United Kingdom

\section{OPEN ACCESS}

Edited by:

Sascha Thewes,

Freie Universität Berlin, Germany

Reviewed by:

Charley Christian Staats,

Federal University of Rio Grande do

Sul (UFRGS), Brazil

Sascha Brunke,

Leibniz-Institut für

Naturstoff-Forschung und

Infektionsbiologie and Hans Knöll

Institut, Germany

*Correspondence:

Jason S. King

jason.king@sheffield.ac.uk

Simon A. Johnston

s.a.johnston@sheffield.ac.uk

these authors have contributed equally to this work.

Received: 24 November 2017 Accepted: 19 March 2018 Published: 09 April 2018

Citation:

Watkins RA, Andrews A, Wynn C, Barisch C, King JS and Johnston SA

(2018) Cryptococcus neoformans Escape From Dictyostelium Amoeba by Both WASH-Mediated Constitutive

Exocytosis and Vomocytosis.

Front. Cell. Infect. Microbiol. 8:108.

doi: 10.3389/fcimb.2018.00108
Cryptococcus neoformans is an environmental yeast that can cause opportunistic infections in humans. As infecting animals does not form part of its normal life-cycle, it has been proposed that the virulence traits that allow cryptococci to resist immune cells were selected through interactions with environmental phagocytes such as amoebae. Here, we investigate the interactions between C. neoformans and the social amoeba Dictyostelium discoideum. We show that like macrophages, D. discoideum is unable to kill $C$. neoformans upon phagocytosis. Despite this, we find that the yeast pass through the amoebae with an apparently normal phagocytic transit and are released alive by constitutive exocytosis after $\sim 80 \mathrm{~min}$. This is the canonical pathway in amoebae, used to dispose of indigestible material after nutrient extraction. Surprisingly however, we show that upon either genetic or pharmacological blockage of constitutive exocytosis, $C$. neoformans still escape from $D$. discoideum by a secondary mechanism. We demonstrate that constitutive exocytosis-independent egress is stochastic and actin-independent. This strongly resembles the non-lytic release of cryptococci by vomocytosis from macrophages, which do not perform constitutive exocytosis and normally retain phagocytosed material. Our data indicate that vomocytosis is functionally redundant for escape from amoebae, which thus may not be the primary driver for its evolutionary selection. Nonetheless, we show that vomocytosis of $C$. neoformans is mechanistically conserved in hosts ranging from amoebae to man, providing new avenues to understand this poorly-understood but important virulence mechanism.

Keywords: cryptococcus, Dictyostelium, amoeba, pathogen, exocytosis, cryptococcosis, vomocytosis, WASH

\section{INTRODUCTION}

Cryptococcus neoformans is a basidiomycete yeast found globally in a wide variety of natural environments. Unusually for an environmental yeast, C. neoformans is also a pathogen of animals. Most significant is the fatal infection of the severely immunocompromised, with cryptococcal meningitis caused by C. neoformans responsible for 15\% of AIDS-related deaths (Rajasingham et al., 2017). The interaction of C. neoformans with its host is highly complex, and what differentiates normal immunity from the development of life 
threatening cryptococcal meningitis is well defined (Tenforde et al., 2017).

Macrophages have been repeatedly demonstrated to be critical for protection against $C$. neoformans infection. However, macrophages may also have a role in pathogenesis in the immunocompromised as cryptococci are able to grow and survive within macrophages and may use macrophages as a Trojan horse to disseminate from the lung. In vitro, almost every aspect of macrophage antimicrobial activity is either avoided or manipulated by C. neoformans, which are able to survive and replicate intracellularly, following uptake by phagocytosis (Johnston and May, 2013; Ballou and Johnston, 2017). Survival traits include the generation of a characteristic polysaccharide capsule, which is both anti-phagocytic and helps protect the yeast from the host antimicrobial machinery if it is engulfed, as well as melanin production which serves as a potent antioxidant-protecting the yeast from the phagosomal oxidative attack and the immunomodulatory activity of cell wall chitin (Casadevall et al., 2000; Nosanchuk and Casadevall, 2006; Wiesner et al., 2015).

An additional pathogenic mechanism is the remarkable ability of C. neoformans to promote its non-lytic expulsion from host cells in a process known as vomocytosis (Alvarez and Casadevall, 2006; Ma et al., 2006). This enables the yeast to escape whilst leaving the host phagocyte intact, thus preventing immune stimulation and promoting dissemination. Whilst it has been shown that vomocytosis is suppressed by host actin polymerization (Johnston and May, 2010) and can be modulated by host Annexin A2 and Mitogen Activated Protein kinase (ERK5) activity (Stukes et al., 2016; Gilbert et al., 2017) little is known of the underlying molecular mechanisms underlying expulsion. Nonetheless, vomocytosis has been observed in both cell culture and in vivo models and is thought to significantly contribute to C. neoformans virulence (Alvarez and Casadevall, 2006; Ma et al., 2006; Bojarczuk et al., 2016; Johnston et al., 2016; Gilbert et al., 2017).

As with other opportunistic pathogens, it is unlikely that interactions with mammalian macrophages have been the evolutionary drivers of C. neoformans virulence. Cryptococci are free-living fungi with a life cycle that is not dependent on infecting an animal host. It has therefore been proposed that the mechanisms that allow $C$. neoformans to survive and grow in macrophages have primarily evolved to avoid predation by phagocytes in its natural environment, such as amoebae (Steenbergen et al., 2001; Casadevall, 2012; Watkins et al., 2017).

Like leukocytes, amoebae are professional phagocytes, using their chemotactic and phagocytic abilities to capture and kill environmental microbes for food. Despite the large evolutionary distance between them, much of the machinery and mechanisms for phagocytosis and phagosome maturation are highly conserved between amoebae and mammalian immune cells (Boulais et al., 2010). Traits that have evolved to help yeast and bacteria avoid being killed by amoebae in the environment are therefore likely to have similar effects when they encounter mammalian immune cells.
Previous studies have demonstrated similarities in the interactions between $C$. neoformans with amoebae and macrophages. C. neoformans is able to both survive phagocytosis and replicate intracellularly within Acanthamoeba castellanii, ultimately being released alive without causing lysis of the host amoeba (Steenbergen et al., 2001; Chrisman et al., 2010). Due to its amenability to genetic manipulation, the social amoeba Dictyostelium discoideum has been used a model host for a number of human pathogens and is also susceptible to C. neoformans infection (Steenbergen et al., 2003). Importantly, passage through $D$. discoideum caused a stimulation in C. neoformans capsule expansion and melanization together with a corresponding increase in subsequent virulence in mice (Steenbergen et al., 2003). Interactions with amoebae can therefore directly influence interactions between C. neoformans and mammalian immune cells.

The fate of internalized material in animal cells is variable and complex. There are examples of the expulsion of internalized material from a variety of cell types, particularly in the context of antigen presentation (Chen and Jondal, 2004; Peters et al., 2006; Griffiths et al., 2012; Le Roux et al., 2012; Turner et al., 2016). However, animal macrophages (notably tissue resident cells, such as alveolar macrophage) have the ability to retain particulate matter that may otherwise be damaging (Bai et al., 2015). In contrast, the constitutive exocytosis of phagocytosed material by amoeba has been demonstrated in diverse species including D. discoideum, Amoeba proteus, Entamoeba histolytica and A. castellanii (Weisman and Korn, 1967; Ravdin et al., 1988; Christofidou-Solomidou and Stockem, 1992; Clarke et al., 2010) and thus appears to be a necessary and general feature of freeliving amoebae. Therefore macrophages and amoebae differ in their retention of phagocytosed material.

Recently it was shown that constitutive exocytosis in D. discoideum is dependent on the activity of the WASH (WASP And SCAR Homolog) complex (Carnell et al., 2011). WASH is a direct activator of the ARP2/3 (Actin Related Protein 2/3) complex, causing the polymerization of actin on the surface of vesicles and driving membrane protein sorting and recycling (Derivery et al., 2009; Gomez and Billadeau, 2009; Zech et al., 2011; Seaman et al., 2013). Whilst an early phase of WASH activity drives the retrieval of cell surface proteins from phagosomes (Buckley et al., 2016), a second phase of activity occurs after $40-60 \mathrm{~min}$ of digestion, driving the removal of the vacuolar (V)-ATPase and phagosomal neutralization. This facilitates hydrolase retrieval (King et al., 2013) and is a prerequisite for exocytosis. Consequently $D$. discoideum cells lacking WASH have a complete block in constitutive exocytosis (Carnell et al., 2011).

As constitutive exocytosis represents the normal mechanism of non-lytic release of phagosomal contents, we hypothesized that this may be involved in the escape of live C. neoformans from amoebae. We show that $C$. neoformans survive phagocytosis by $D$. discoideum, but follow an apparently normal phagosomal transit and are normally released alive by WASH-dependent constitutive exocytosis. However, when constitutive exocytosis is blocked, C. neoformans still escape in a WASH and actinindependent manner reminiscent of vomocytosis. This 
demonstrates redundant, mechanistically different egress mechanisms with implications for the understanding of the evolutionary drivers of cryptococcal virulence.

\section{MATERIALS AND METHODS}

\section{Strains and Cell Culture}

For all experiments the Ax2 axenic strain of $D$. discoideum was used, both as "wild type" as well as the parental of the previously published WASH-mutant strain (Carnell et al., 2011). D. discoideum were cultured in filter-sterilized HL-5 medium (Formedium, Norfolk, UK) at $22^{\circ} \mathrm{C}$. Cells expressing GFP fused to the vatM subunit of the vacuolar-ATPase were generated using plasmid pMJC25 (Carnell et al., 2011).

Unless otherwise stated, C. neoformans var. grubii (serotype A) strain H99 $\alpha$ stably expressing mCherry was used (Gibson et al., in review). The previously published plb1 and cap59 mutants were generated from the alternative wild-type parent H99, which was used as control when appropriate (Chen et al., 2000; Voelz et al., 2010). C. neoformans were grown in YPD medium at $28^{\circ} \mathrm{C}$ prior to experiments, but washed and resuspended in HL5 medium before infecting D. discoideum. $C$. neoformans were heat-killed by incubation at $65^{\circ} \mathrm{C}$ for $30 \mathrm{~min}$, before washing in PBS. UV-killing was performed by exposure of C. neoformans cultures to $4 \mathrm{~J}$ of UV using a UVIlink CL-5087 cross-linker illuminator. Killing was always confirmed by plating samples on YPD agar to check absence of growth.

\section{Infections and Microscopy}

Prior to imaging, $2 \times 10^{6} \mathrm{D}$. discoideum cells were seeded in $2 \mathrm{ml}$ HL5 medium in glass-bottom $35 \mathrm{~mm}$ dishes (Mat-Tek), left to adhere for $30 \mathrm{~min}$. Then $2 \times 10^{5} \mathrm{C}$. neoformans cells, $4.5 \mu \mathrm{m}$ green fluorescent YG unmodified beads (Polysciences Inc., Pennsylvania, USA), or TRITC-labeled heat killed S. cerevisiae (kind gift from Thierry Soldati; University of Geneva) were added prior to imaging. This differed for latrunculin treatment experiments: amoebae were mixed with yeast at a ratio of $1: 1$ and left for $1 \mathrm{~h}$ for phagocytosis to occur before addition of $5 \mu \mathrm{m}$ Latrunculin A (Cayman chemical Co.).

Long term time-lapse movies were recorded using a Nikon TI- E with a CFI Plan Apochromat $\lambda$ 20x N.A.0.75 at $22^{\circ} \mathrm{C}$. Images were captured on a large-format Andor Neo $5.5 \mathrm{~s}$ CMOS camera for $12 \mathrm{~h}$, imaging 4 fields of view every $30 \mathrm{~s}$. Transit times were determined as the time from phagocytosis to release. Events where cells could not be tracked for a minimum of $500 \mathrm{~min}$ post-phagocytosis were excluded from the analysis.

Spinning disc microscopy was performed on a Perkin-Elmer Ultraview VoX spinning disk confocal microscope running on an Olympus $1 \times 81$ body with an UplanSApo 60x oil immersion objective (NA 1.4). Images were captured on a Hamamatsu C9100-50 EM-CCD camera.

\section{Statistical Analysis}

Mann Whitney test was used to test significance between continuous data and Fisher's exact test for categorical data. $P$-values below 0.05 (with modification for multiplicity of testing) were considered statistically significant. All statistical tests were performed with GraphPad Prism version 7.

\section{RESULTS}

\section{Cryptococcus neoformans Is Constitutively Exocytosed by Amoebae}

To determine whether $C$. neoformans utilizes a pathogen-specific mechanism of release or follows the normal phagocytic pathway of engulfment and constitutive exocytosis in amoeba we first compared the transit of $C$. neoformans with that of inert particles.

Using time lapse microscopy, we followed the phagocytosis and release of $C$. neoformans compared to heat killed nonpathogenic yeast Saccharomyces cerevisiae and $4.0 \mu \mathrm{m}$ latex beads by $D$. discoideum. All three cargoes were of a similar size (between 3 and $5 \mu \mathrm{m}$ ) and all were exocytosed from amoebae (Figures 1A-C). However, unlike the vomocytosis of C. neoformans from macrophages which happens stochastically and inefficiently over a period of many hours (Ma et al., 2006; Johnston and May, 2010), 100\% of phagosomes fused with the plasma membrane, exocytosing their contents within $4 \mathrm{~h}$ of engulfment in all cases.

Surprisingly, whilst we attempted to use killed C. neoformans as controls, both heat- or UV-killing of the yeast reduced phagocytosis by $D$. discoideum by $90 \%$ (Figure $1 \mathbf{H}$ ). This effect was further compounded in WASH-null amoebae, in which phagocytosis of UV-killed C. neoformans was never observed in 3 independent experiments, consistent with previous reports of phagocytosis defects in these mutants due to reduced surface levels of phagocytic receptors (Buckley et al., 2016). The reason why killed C. neoformans resist phagocytosis is unclear, but it was not possible to observe sufficient events for analysis and therefore heat-killed S. cerevisiae were used as non-pathogenic controls in subsequent experiments.

From the time-lapse movies, we were able to define precise transit times for phagosomes containing the different cargoes, tracking individual particles from engulfment to release (Figures 1D-F). Comparison of the transit time demonstrated that both fungal cells took slightly longer to complete the phagocytic cycle than latex beads however, there was no significant difference between heat killed $S$. cerevisiae and C. neoformans (median transit time latex beads $=61 \mathrm{~min}$, heat killed $S$. cerevisiae $=82 \mathrm{~min}$ and $C$. neoformans $=84 \mathrm{~min}$, Figure 1G). Importantly the $C$. neoformans were able to resist killing by the amoebae and were exocytosed alive, as indicated by subsequent budding and dividing after egress consistent with previous studies (Steenbergen et al., 2003). Therefore, despite their ability to survive phagocytosis by $D$. discoideum, transit time of $C$. neoformans is indistinguishable from normal constitutive exocytosis.

\section{The V-ATPase Is Rapidly Recruited to Phagosomes Containing C. neoformans and Removed Prior to Exocytosis}

Unlike the vomocytosis of cryptococci, a number of molecular requirements for constitutive exocytosis in amoebae have 


\section{A}

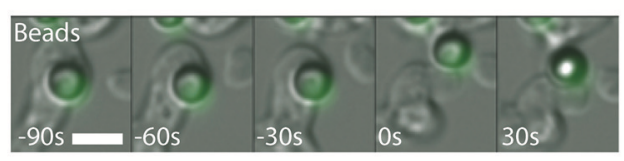

B

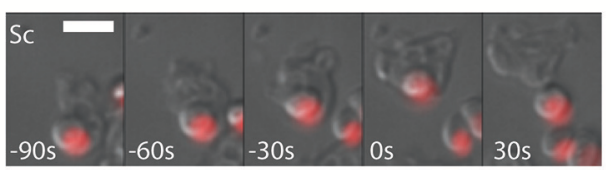

C

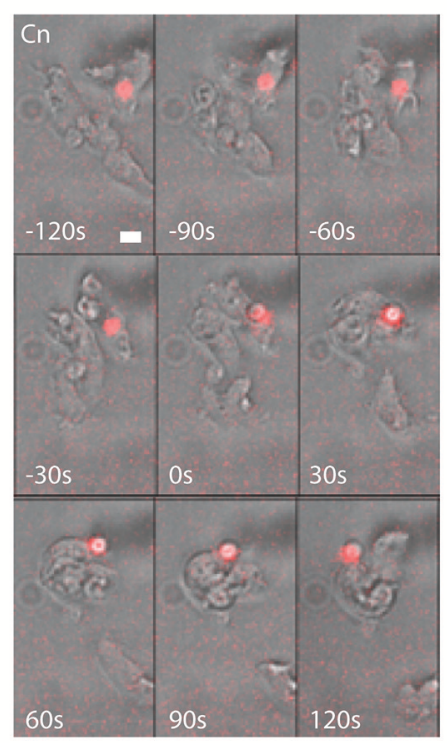

G

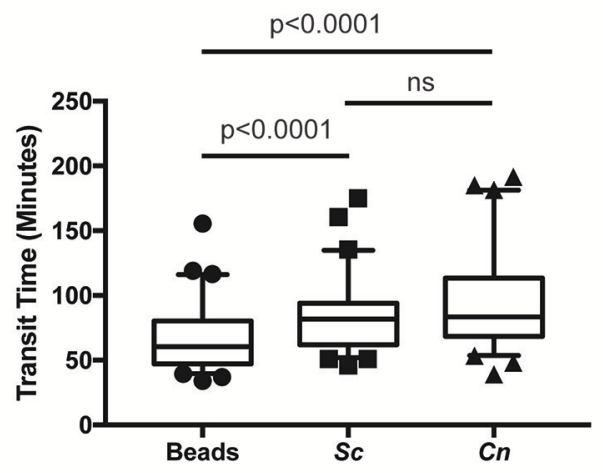

D

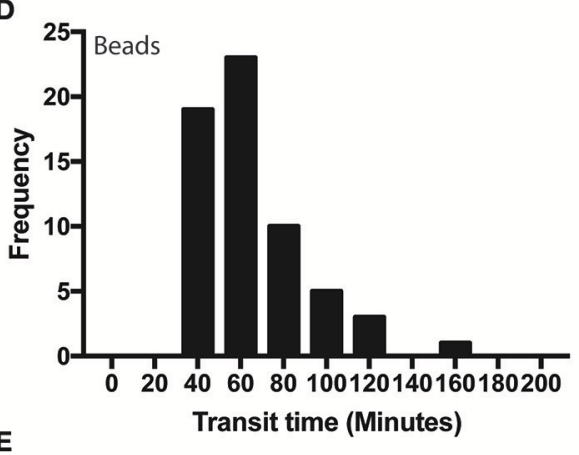

E

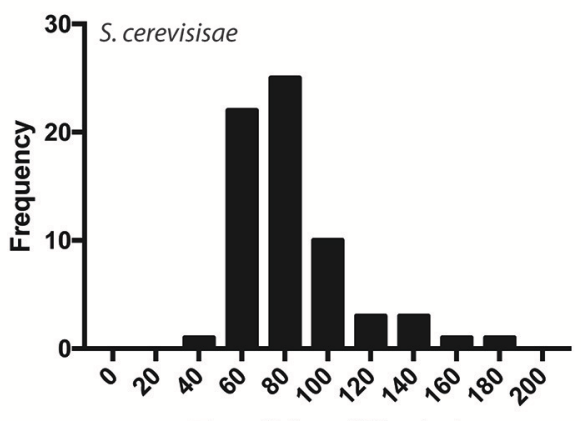

Transit time (Minutes)

$\mathbf{F}$

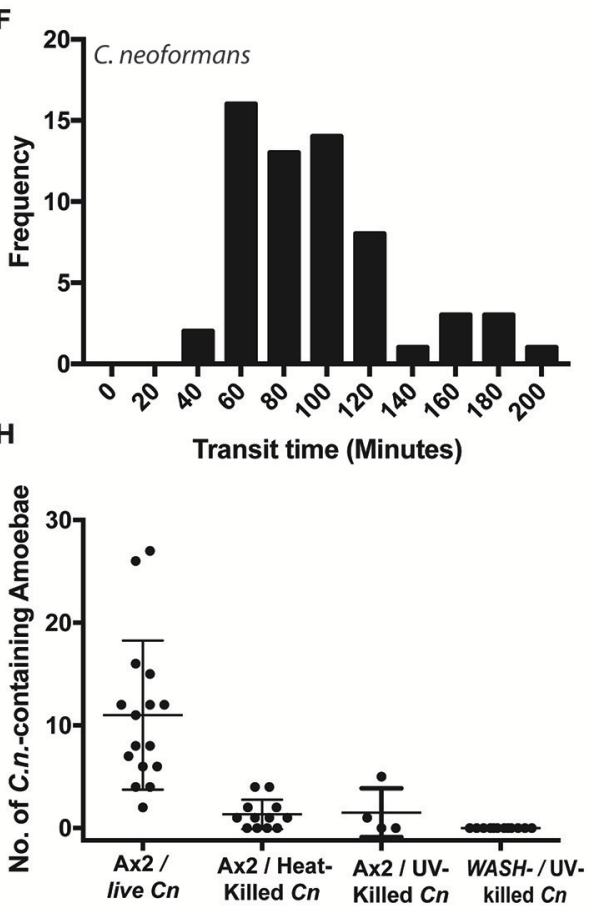

FIGURE 1 | Cryptococcus neoformans is constitutively exocytosed from Dictyostelium discoideum amoeba. (A-C) Example exocytosis of (A) 4.5 um green fluorescent latex beads (images start at 40 min after phagocytosis) (B) heat killed Saccharomyces cerevisiae (images start at 60 min after phagocytosis) and (C) C. neoformans strain Kn99mCherry from wild type D. discoideum strain Ax2 (images start at 60 min after phagocytosis). Time 0 s indicates point of exocytosis. Scale bars $5 \mu \mathrm{m}$. (D-F) Frequency histograms of combined transit times measured from three independent $12 \mathrm{~h}$ time lapses. (D) Latex beads 126 transit times. (E) Heat killed S. cerevisiae 66 transit times. (F) C. neoformans 57 transit times. (G) Comparison of transit times for latex beads, heat killed S. cerevisiae and C. neoformans. (H) Quantification of phagocytic events for live, heat killed and UV killed cryptococci in Ax2 and WASH null cells. P-values are Mann-Whitney test. 
been identified. We therefore next asked whether passage of $C$. neoformans through $D$. discoideum followed the normal path of maturation and constitutive exocytosis.

During normal transit, phagosomes rapidly accumulate the VATPase and acidify within 2-3 min of engulfment; the V-ATPase is subsequently retained for $\sim 45 \mathrm{~min}$ to allow digestion before retrieval and phagolysosomal neutralization prior to constitutive exocytosis (Clarke et al., 2002, 2010). Using cells expressing GFP-fused to the VatM subunit of the V-ATPase, we monitored recruitment to phagosomes following engulfment of cryptococci. $\mathrm{V}$-ATPase was present, on average, within $114 \pm 44 \mathrm{~s}$ (Figure 2A, S.D., $n=6$ ) of phagocytosis consistent with published data for inert phagosomes (Clarke et al., 2002; Buckley et al., 2016). To test if V-ATPase recruitment was maintained, we also measured the proportion of $C$. neoformans-containing phagosomes positive for GFP-VatM after $20 \mathrm{~min}$ incubation of cryptococci with amoebae-before any post-lysosomal transitions should have occurred (Figure 1F). V-ATPase was clearly visible on $90.0 \%$ of phagosomes (68/76 from three independent experiments). However, when we looked after $1 \mathrm{~h}$, we were able to observe recycling of the V-ATPase from live (budding) C. neoformanscontaining phagosomes prior to exocytosis (Figure 2B). Both phagosomal transit time and V-ATPase dynamics are therefore unaffected by pathogenic C. neoformans indicating that the normal mode of release is through canonical constitutive exocytosis.

\section{C. neoformans Can Escape From Amoebae by Both WASH-Dependent and -Independent Mechanisms}

To test the hypothesis that Cryptococci-containing phagosomes are normally expelled through the constitutive exocytosis pathway, we investigated exocytosis in WASH-null cells. In D. discoideum, WASH is essential for V-ATPase recycling and constitutive exocytosis, allowing us to specifically genetically ablate this pathway (Carnell et al., 2011).

In agreement with previous studies we found that phagosomes containing heat killed $S$. cerevisiae were never released from $W A S H$-null amoebae within our $12 \mathrm{~h}$ period of observation (Figure 3A). In contrast, phagosomes containing C. neoformans were still exocytosed but with significantly altered dynamics (Figure 3B). Whilst $>90 \%$ of C. neoformans-containing phagosomes are exocytosed within $2 \mathrm{~h}$ in wild type amoebae (Figure 1F), release from $W A S H$-null cells was much more variable with between 20 and $60 \%$ escaping over $12 \mathrm{~h}$ (Figure 3C). Cryptococcus-containing phagosome transit was much slower through $W A S H$-null cells with very little overlap with the exocytosis from wild-type amoebae (ca. Figures 1F, 3C) and was much less synchronous, appearing to occur stochastically any time from 3 to $>10$ h (Figure 3D). Notably, both this variation and timing is comparable to that reported for vomocytosis of cryptococci from animal cells (Johnston and May, 2010).

The actin cytoskeleton is a negative regulator of vomocytosis in mammals, but essential for constitutive phagosome exocytosis in D. discoideum (Ma et al., 2006; Carnell et al., 2011). Therefore, we predicted that inhibition of actin polymerization would copy the phenotype of WASH-null Dictyostelium for both heat killed $S$. cerevisiae and $C$. neoformans containing phagosomes. When we measured the percentage exocytosis of heat killed S. cerevisiae containing phagosomes we found that while $100 \%$ of phagosomes were exocytosed by untreated amoebae we only observed a single exocytosis event out of 90 phagosomes analyzed over $12 \mathrm{~h}$ when actin polymerization was blocked by latrunculin A treatment after phagocytosis (Figure 3E). In contrast, $\sim 20 \%$ of $C$. neoformans-containing phagosomes in latrunculin A-treated amoebae were released over the same period (Figure 3F). Transit times were again significantly longer than with untreated amoebae, and were indistinguishable to the phenotype observed with WASH-null amoebae (Figure 3G). Thus, whilst cryptococci are normally released by constitutive exocytosis from $D$. discoideum, they can also escape by a mechanistically different route upon either pharmacological or genetic blockade of the constitutive pathway, highly reminiscent of vomocytosis.

\section{C. neoformans Mutants cap59 and plb1 Do Not Exhibit Defects in Vomocytosis in Amoebae}

We next tested whether cryptococcal virulence factors that affect vomocytosis from mammalian cells play conserved roles in egress from $D$. discoideum. C. neoformans mutants with defects in polysaccharide capsule formation or deletion of the phospholipase PLB1 both exhibit reduced rates of vomocytosis and pathogenicity in animal cells (Cox et al., 2001; Noverr et al., 2003; Chayakulkeeree et al., 2011; Evans et al., 2015). However, when we measured the rates of release of acapsular cap59 and plb1 mutant strains from wild-type D. discoideum we found that release of both mutants was unaffected: there were no significant differences in either frequency or transit time compared to the parental C. neoformans strain (H99) both with and without blockage of the constitutive pathway with latrunculin A (Figures 4A,B). This was an intriguing finding, suggesting differences in the signaling pathways to vomocytosis between amoebae and macrophages but a conservation of molecular mechanism.

\section{In the Absence of Constitutive Exocytosis C. neoformans Can Persist and Grow Intracellularly}

Constitutive exocytosis of indigestible phagosomal material is critical for organisms that rely on phagocytosis for nutrition. Furthermore, amoebae may ingest microbes such as cryptococci that are able to actively resist phagosomal killing. Therefore, we investigated if, in the absence of constitutive exocytosis, cryptococci posed a greater threat to amoebae.

We observed no cell lysis of either wild-type or WASH-null cells infected with $C$. neoformans as indicated by the continued motility of the amoebae throughout the phagocytic cycle and after fungi egress. Low levels of amoeba lysis were observed upon latrunculin A treatment, most likely due to the severe effects of complete actin depolymerization. Killing and digestion 
A

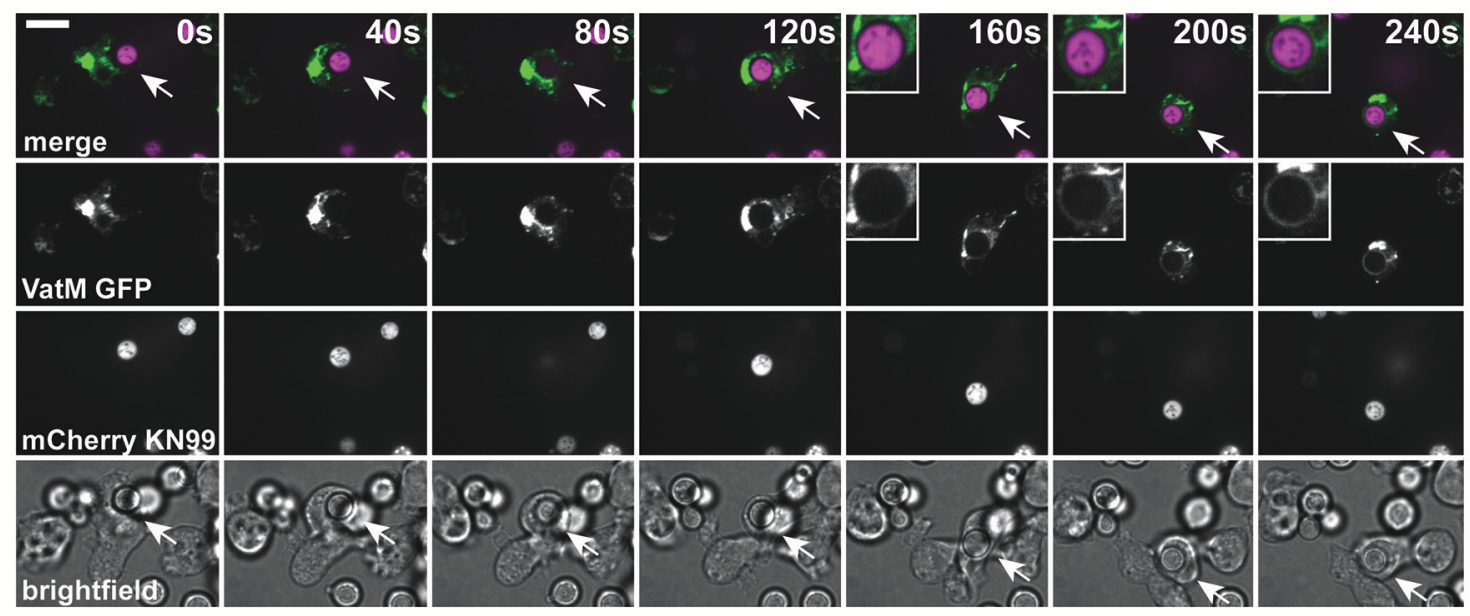

B

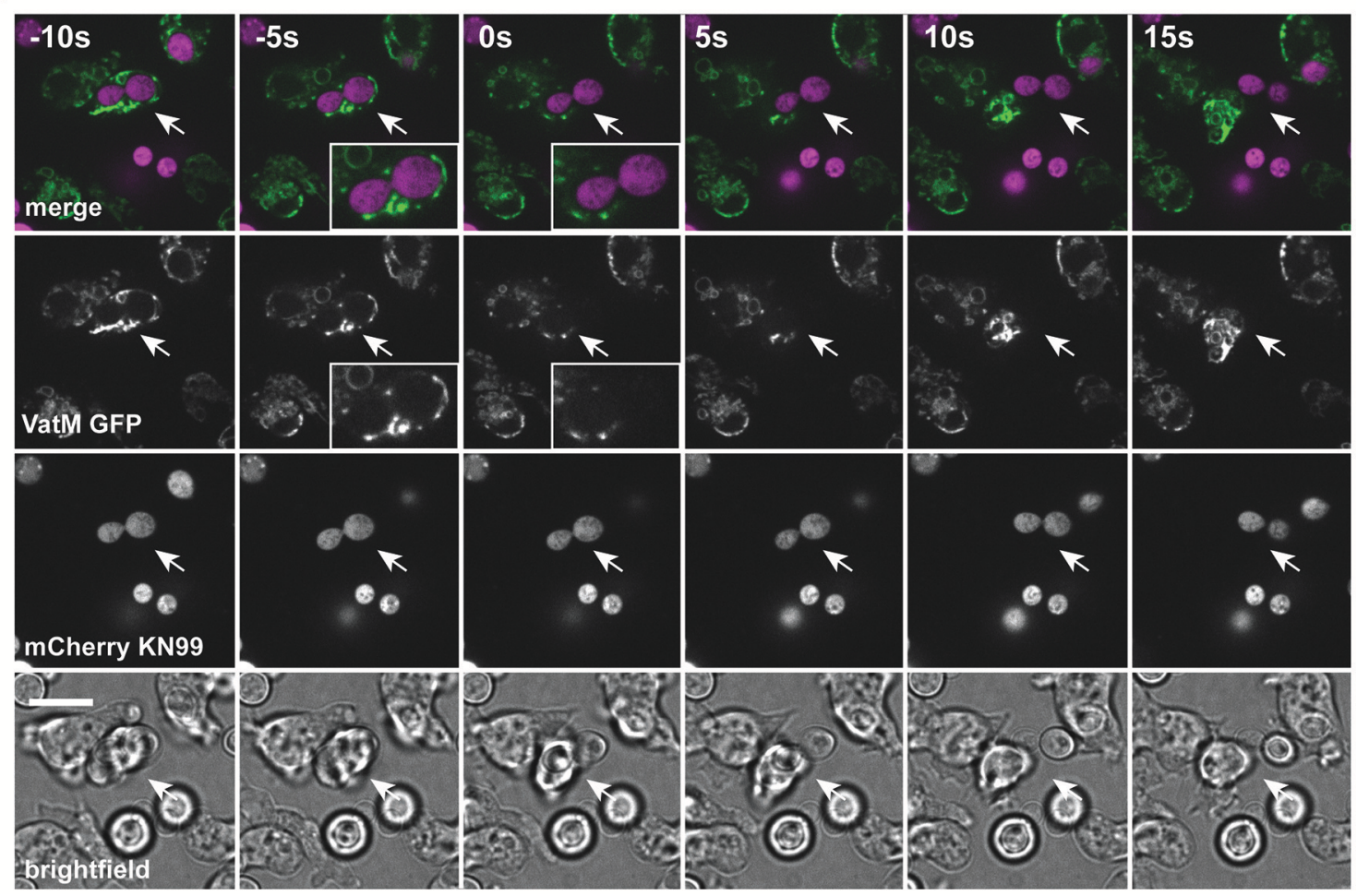

FIGURE 2 | Phagosomes containing Cryptococcus neoformans acquire V-ATPase following phagocytosis that is lost prior to exocytosis. (A) Confocal time lapse microscopy of $C$. neoformans phagocytosis by wild type $D$. discoideum strain $A \times 2$. Representative time lapse from three independent experiments. Images were captured every $10 \mathrm{~s}$. VatM is a subunit of the $D$. discoideum V-ATPase complex. Arrow indicates phagocytosed cryptococcal cell. Inset box is a magnification of phagosome containing cryptococcal cell demonstrating acquisition of V-ATPase. (B) Confocal time lapse microscopy of C. neoformans exocytosis by wild type $D$. discoideum strain Ax2. Representative time lapse from three independent experiments. Images were captured every $5 \mathrm{~s}$. Arrow indicates exocytosed cryptococcal cell. Inset box is a magnification of exocytosed cryptococcal cell demonstrating absence of V-ATPase prior to exocytosis. Scale bars $10 \mu \mathrm{m}$.

of yeast is indicated by the transformation of the phagosome to a granular and irregular shape, whereas growth can be inferred from yeast budding. When constitutive exocytosis was blocked with latrunculin A on average, more than $80 \%$ of cryptococci persisted within Dictyostelium phagosomes without signs of death and digestion, with $13 \%$ actively budding over the $12 \mathrm{~h}$ of the experiment (Figures 5A,B). In this respect the plb1 mutant behaved similarly to wild type cryptococci. The acapsular strain cap59 was phagocytosed twice as efficiently as the wild type cryptococcus strain (11 internalized cap59 cryptococci per 100 
A

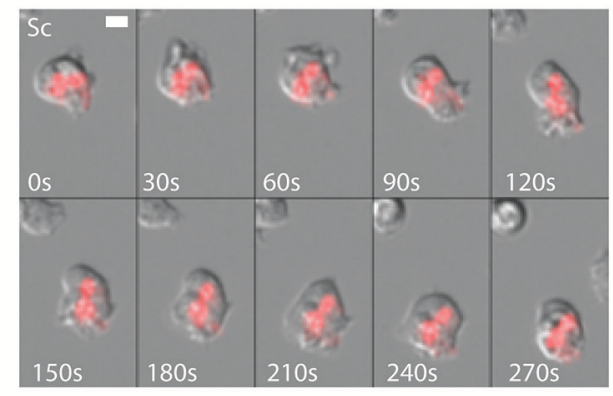

B

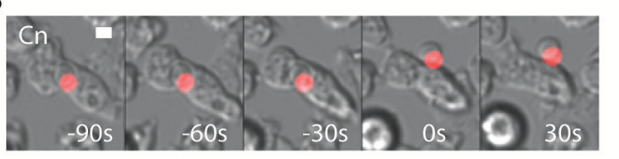

C

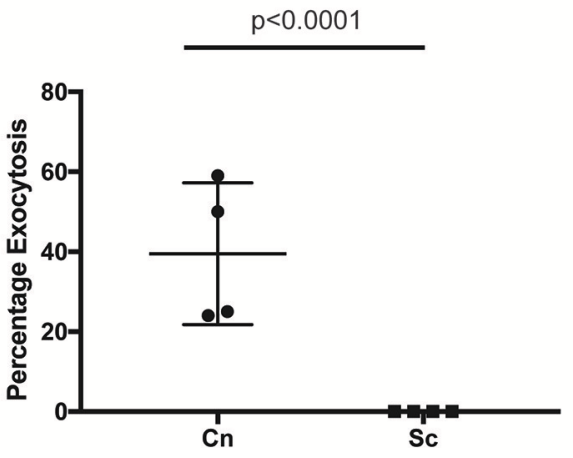

D

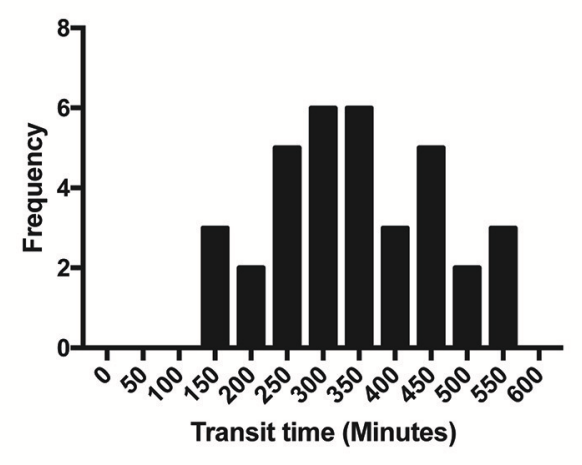

$\mathbf{E}$

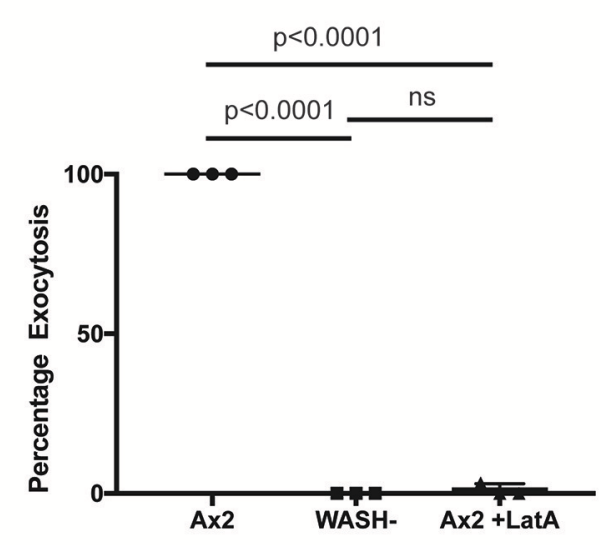

$\mathbf{F}$

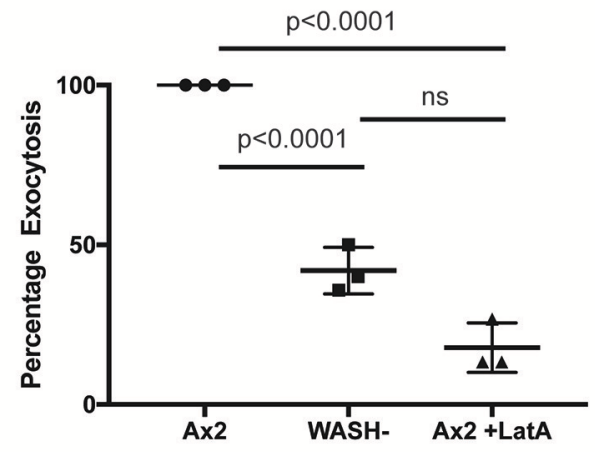

G

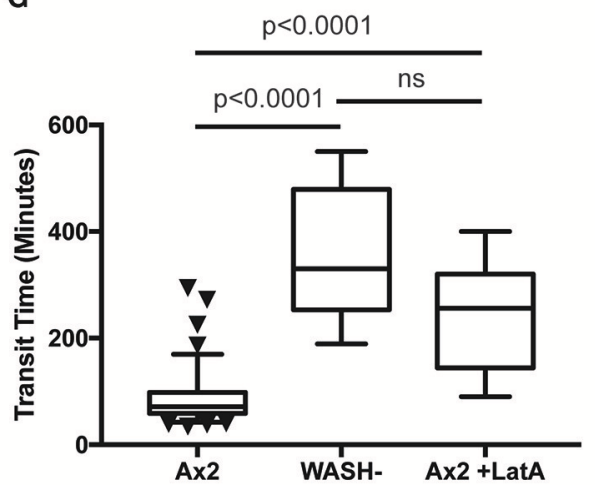

FIGURE 3 | Exocytosis of Cryptococcus neoformans from Dictyostelium is dependent on WASH and the actin cytoskeleton. (A) Heat killed S. cerevisiae are not exocytosed from WASH null $D$. discoideum. Example from $12 \mathrm{~h}$ time lapse imaging of heat killed $S$. cerevisiae in WASH null $D$. discoideum representative of 60 phagosomes containing heat killed S. cerevisiae from three independent experiments (images start at 500 min after phagocytosis). (B) $C$. neoformans are exocytosed from WASH null $D$. discoideum. Example from $12 \mathrm{~h}$ time lapse imaging representative of 62 phagosomes containing $C$. neoformans from three independent experiments (images start at $200 \mathrm{~min}$ after phagocytosis). Scale bars $5 \mu \mathrm{m}$. (C) Quantification of the percentage of exocytosis of $C$. neoformans and heat killed S. cerevisiae from WASH null D. discoideum from three independent $12 \mathrm{~h}$ time lapses. (D) Frequency histogram of combined 35 transit times measured from three independent $12 \mathrm{~h}$ time lapses. (E) Exocytosis of heat killed S. cerevisiae from wild type Ax2 but not WASH null or latrunculin A treated Ax2 D. discoideum.

Quantification of the percentage of exocytosis from three independent $12 \mathrm{~h}$ time lapses. Total of 60 phagosomes were analyzed from each condition. $P$-values are Fishers test. (F) Exocytosis of $C$. neoformans from wild type Ax2, WASH null, and latrunculin A treated $D$. discoideum. Quantification of the percentage of exocytosis from three independent $12 \mathrm{~h}$ time lapses. Total of 60 phagosomes were analyzed from each condition. $P$-values are Fishers test. (G) Transit times of $C$. neoformans through WASH null and latrunculin A treated Ax2 D. discoideum are not significantly different. $P$-values are Mann-Whitney test.

amoeba vs. 5.3 cryptococci per 100 amoeba, 300 amoeba analyzed from three independent experiments). However, the acapsular cap59 strain appeared to be growth-arrested within $D$. discoideum phagosomes, as budding was never observed (0/90 phagosomes,
$P=0.0011$ compared to H99, Fishers test; Figure 5B). Whilst this is consistent with other studies (Feldmesser et al., 2000; Steenbergen et al., 2001) surprisingly, we also never observed the collapse of cap59-containing phagosomes within the $12 \mathrm{~h}$ 


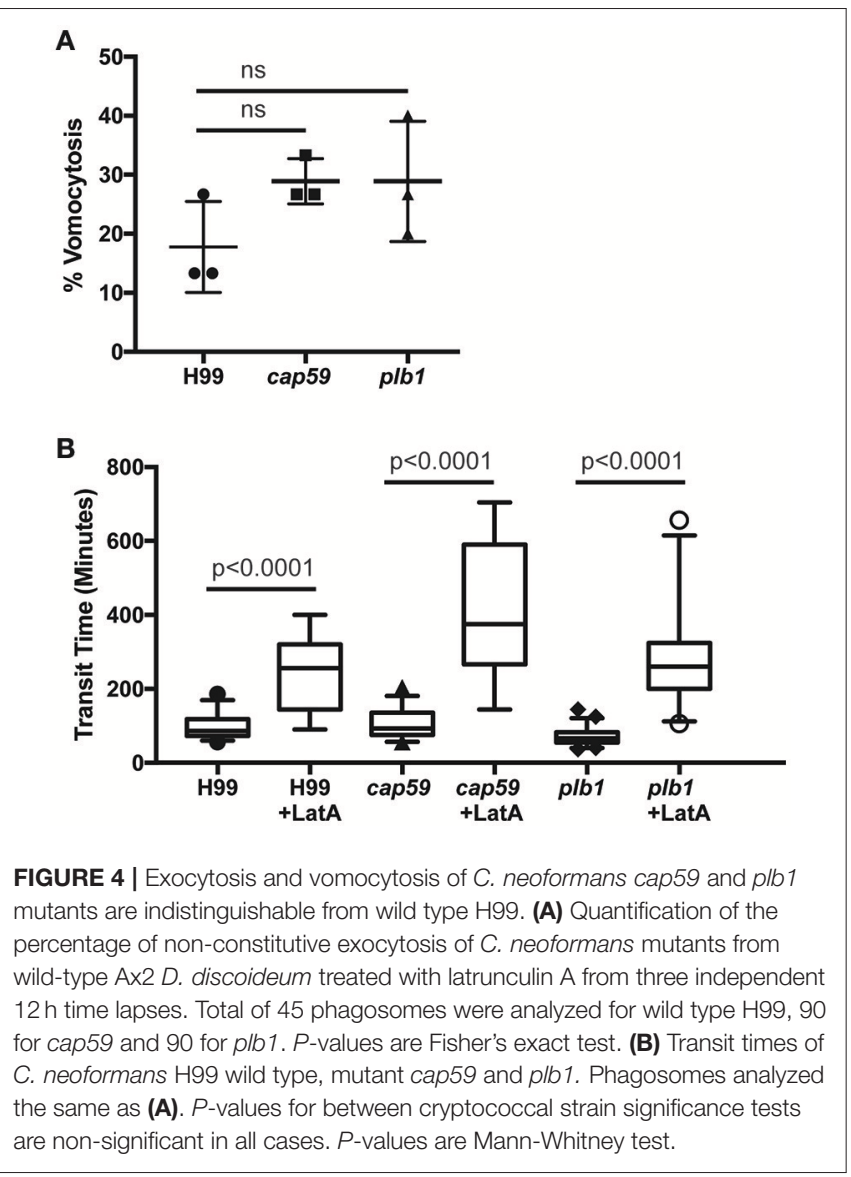

indicating yeast death. This implies that either acapsular cells survive under growth arrest, or that death of cap59 cells occurs over a longer period than we are able to observe in this assay. Nonetheless, in the absence of constitutive exocytosis, yeast are able to replicate intracellularly within the retained phagosomes.

\section{DISCUSSION}

In this work we have investigated the interactions between the environmental yeast $C$. neoformans and an environmental amoeba, D. discoideum. As C. neoformans can evade human immune cells and cause opportunistic infection in immunocompromised individuals we sought to test the hypothesis that the yeast virulence mechanisms had evolved to protect against amoebae in the environment.

In agreement with previous reports (Steenbergen et al., 2003), we found that $C$. neoformans were almost completely impervious to predation by $D$. discoideum; we consistently observed lower rates of C. neoformans phagocytosis compared to other particles, and even when the yeast were engulfed the amoebae were unable to kill them. This is similar to the interactions with mammalian macrophages, indicating that C. neoformans employs similar phagocyte evasion strategies. Chief amongst these is the characteristic cryptococcal capsule
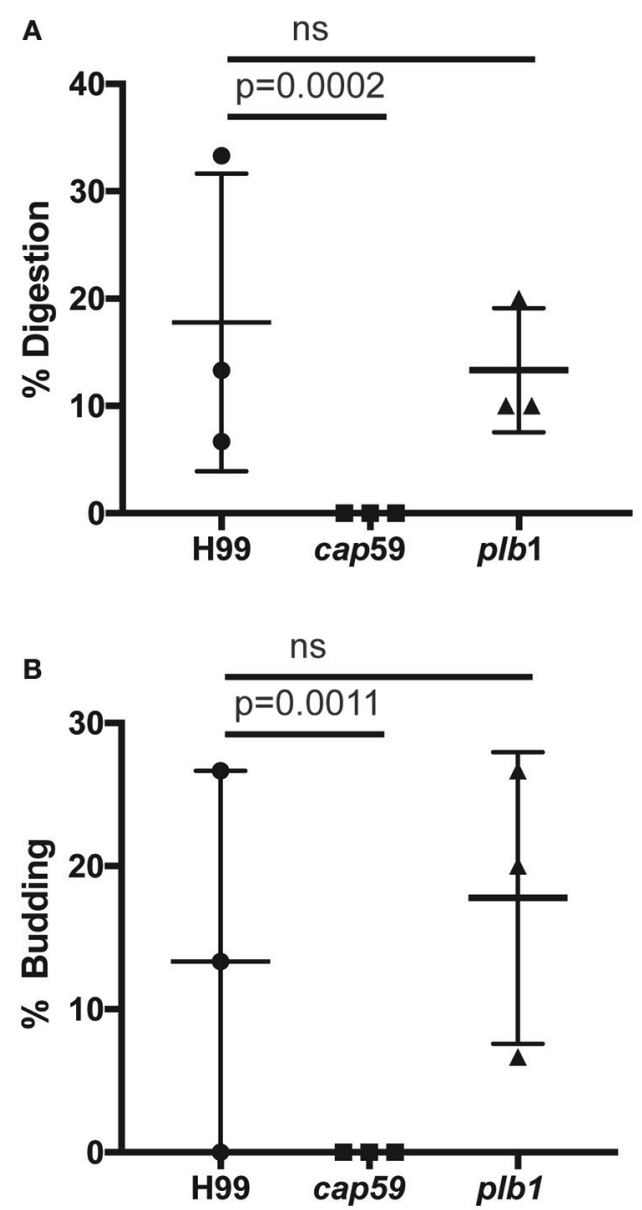

FIGURE 5 | Outcomes of interaction between cryptococcal cells and amoebae with inhibition of constitutive exocytosis. Quantification of outcome from three independent $12 \mathrm{~h}$ time lapses. Numbers of phagosomes analyzed the same as Figure 4A. (A) Percentage of fungal cells digested by amoebae over $12 \mathrm{~h}$. (B) Percentage of fungal cells that budded while intracellular in amoebae over $12 \mathrm{~h}$. $P$-values are Fisher's exact test.

which is highly anti-phagocytic and also provides protection from oxidative attack. Consistent with this both we, and others found capsule deficient yeasts were unable to grow within amoebae (Steenbergen et al., 2001, 2003) and we found that the acapsular strain was taken up twice as frequently as the wild type.

Whilst C. neoformans typically persist and proliferate in macrophages for many hours before escaping by vomocytosis, we found they passed through $D$. discoideum via an apparently normal phagosomal transit in just $\sim 80 \mathrm{~min}$. This short time period provides limited opportunity for intracellular growth of fungal cells or the lysis of amoebae. Whilst others have shown that $C$. neoformans are able to grow (and therefore extract nutrients) when co-incubated with amoebae over several days (Steenbergen et al., 2001, 2003). The relatively short amount of time spent inside the amoeba implies that much of this growth is extracellular and intracellular growth has not been directly demonstrated by high resolution time lapse imaging (Steinman 
et al., 1976; Derengowski et al., 2013; Fu and Casadevall, 2018). We were unable to identify intracellular proliferation in the absence of the constitutive exocytosis inhibition. It therefore seems unlikely that C. neoformans has successfully evolved mechanisms to support intracellular replication within amoebae that exhibit rapid phagosomal transit, as demonstrated for D. discoideum, Amoeba proteus, Entamoeba histolytica and A. castellanii (Weisman and Korn, 1967; Ravdin et al., 1988; Christofidou-Solomidou and Stockem, 1992; Clarke et al., 2010).

Although D. discoideum are unable to kill phagocytosed C. neoformans the transit time is identical to that of heatkilled non-pathogenic yeast. This suggests that there is no major subversion of normal phagosome maturation. Consistent with this we find that the V-ATPase is both recruited, and retrieved from phagosomes with normal dynamics. This is in contrast to a recent study in macrophages, demonstrating that C. neoformans is able to disrupt phagosome maturation to inhibit acidification and proteolysis to permit intracellular proliferation (Smith et al., 2015). As the crypotococcal-containing phagosome in macrophages is permeabilized shortly after phagocytosis by macrophages (Tucker and Casadevall, 2002) V-ATPase delivery may be intact in both systems, but ineffective due to proton leakage.

Surprisingly, although C. neoformans are released alive from D. discoideum by canonical, WASH-dependent constitutive exocytosis, we found they were still expelled when the constitutive pathway was blocked. This second pathway strongly resembles vomocytosis from macrophages, being non-lytic, stochastic and inhibited by actin (Alvarez and Casadevall, 2006; Ma et al., 2006; Johnston and May, 2010). Vomocytosis remains mechanistically poorly understood and defined, but it seems highly likely that the WASH-independent egress of C. neoformans from $D$. discoideum is an analogous process.

Whilst laboratory strains have mutations that facilitate macropinocytosis and phagocytosis of large particles, wild-type isolates of $D$. discoideum are bacterivores and cannot engulf yeasts (Bloomfield et al., 2015). Therefore although D. discoideum provides a genetically tractable model for amoebae in general, it

\section{REFERENCES}

Alvarez, M., and Casadevall, A. (2006). Phagosome extrusion and host-cell survival after Cryptococcus neoformans phagocytosis by macrophages. Curr. Biol. 16, 2161-2165. doi: 10.1016/j.cub.2006.09.061

Bai, Y., Brugha, R. E., Jacobs, L., Grigg, J., Nawrot, T. S., and Nemery, B. (2015). Carbon loading in airway macrophages as a biomarker for individual exposure to particulate matter air pollution - a critical review. Environ. Int. 74, 32-41. doi: 10.1016/j.envint.2014.09.010

Ballou, E. R., and Johnston, S. A. (2017). The cause and effect of Cryptococcus interactions with the host. Curr. Opin. Microbiol. 40, 88-94. doi: 10.1016/j.mib.2017.10.012

Bloomfield, G., Traynor, D., Sander, S. P., Veltman, D. M., Pachebat, J. A., and Kay, R. R. (2015). Neurofibromin controls macropinocytosis and phagocytosis in Dictyostelium. eLife 4:e04940. doi: 10.7554/eLife.04940

Bojarczuk, A., Miller, K. A., Hotham, R., Lewis, A., Ogryzko, N. V., Kamuyango, A. A., et al. (2016). Cryptococcus neoformans intracellular proliferation and capsule size determines early macrophage control of infection. Sci. Rep. 6:21489. doi: $10.1038 /$ srep 21489 is highly unlikely to be an environmental host for cryptococci. The high phagocytic throughput of amoebae necessitates a mechanism to dispose of indigestible material, and unlike macrophages, there is no advantage in retaining phagosomes indefinitely to restrict an inflammatory response. As constitutive exocytosis appears to be sufficient for successful escape of $C$. neoformans on its own, the evolutionary drivers of a secondary, redundant escape mechanism are unclear. Whether other, more environmentally-relevant amoeba behave differently, or another environmental interaction altogether selects for this virulence trait requires further study.

Whatever the evolutionary basis, we have shown that vomocytosis-like egress is a mechanistically distinct process from constitutive exocytosis and is conserved in hosts from D. discoideum to man. The genetic tractability of Dictyostelium amoebae present an unparalleled opportunity to study the molecular cell biology of host cryptococcal interactions, and the differences in the environmental niche of $C$. neoformans and infection of humans.

\section{AUTHOR CONTRIBUTIONS}

RW, AA, CW, and CB performed experiments and data analysis. Initial concept, funding, experimental design were undertaken by JK and SJ. All authors prepared and edited the manuscript.

\section{ACKNOWLEDGMENTS}

We would like to thank Thierry Soldati for assistance and support developing this project. This work was supported by a Royal Society University Research Fellowship UF140624 to JK, and Medical Research Council and Department for International Development Career Development Award Fellowship MR/J009156/1 to SJ. We are grateful for a Microbiology Society Harry Smith Vacation studentship to support CW. RW was supported by a Bateson Centre BMedSci scholarship. Microscopy studies were supported by an MRC grant (G0700091) and a Wellcome Trust grant (GR077544AIA).

Boulais, J., Trost, M., Landry, C. R., Dieckmann, R., Levy, E. D., Soldati, T., et al. (2010). Molecular characterization of the evolution of phagosomes. Mol. Syst. Biol. 6:423. doi: $10.1038 / \mathrm{msb} .2010 .80$

Buckley, C. M., Gopaldass, N., Bosmani, C., Johnston, S. A., Soldati, T., Insall, R. H., et al. (2016). WASH drives early recycling from macropinosomes and phagosomes to maintain surface phagocytic receptors. Proc. Natl. Acad. Sci. U.S.A. 113, E5906-E5915. doi: 10.1073/pnas.1524532113

Carnell, M., Zech, T., Calaminus, S. D., Ura, S., Hagedorn, M., Johnston, S. A., et al. (2011). Actin polymerization driven by WASH causes V-ATPase retrieval and vesicle neutralization before exocytosis. J. Cell Biol. 193, 831-839. doi: $10.1083 /$ jcb.201009119

Casadevall, A. (2012). Amoeba provide insight into the origin of virulence in pathogenic fungi. Adv. Exp. Med. Biol. 710, 1-10. doi: 10.1007/978-1-4419-5638-5_1

Casadevall, A., Rosas, A. L., and Nosanchuk, J. D. (2000). Melanin and virulence in Cryptococcus neoformans. Curr. Opin. Microbiol. 3, 354-358. doi: 10.1016/S1369-5274(00)00103-X

Chayakulkeeree, M., Johnston, S. A., Oei, J. B., Lev, S., Williamson, P. R., Wilson, C. F., et al. (2011). SEC14 is a specific requirement 
for secretion of phospholipase B1 and pathogenicity of Cryptococcus neoformans. Mol. Microbiol. 80, 1088-1101. doi: 10.1111/j.1365-2958.2011. 07632.x

Chen, L., and Jondal, M. (2004). Alternative processing for MHC class I presentation by immature and CpG-activated dendritic cells. Eur. J. Immunol. 34, 952-960. doi: 10.1002/eji.200324359

Chen, S. C., Wright, L. C., Golding, J. C., and Sorrell, T. C. (2000). Purification and characterization of secretory phospholipase B, lysophospholipase and lysophospholipase/transacylase from a virulent strain of the pathogenic fungus Cryptococcus neoformans. Biochem. J. 347( $\mathrm{Pt} 2), \quad 431-439$. doi: 10.1042/bj3470431

Chrisman, C. J., Alvarez, M., and Casadevall, A. (2010). Phagocytosis of Cryptococcus neoformans by, and nonlytic exocytosis from, Acanthamoeba castellanii. Appl. Environ. Microbiol. 76, 6056-6062. doi: 10.1128/AEM.00812-10

Christofidou-Solomidou, M., and Stockem, W. (1992). Induced pinocytosis and endosomal pathways in Amoeba proteus. Eur. J. Protistol. 28, 56-70. doi: 10.1016/S0932-4739(11)80320-2

Clarke, M., Köhler, J., Arana, Q., Liu, T., Heuser, J., and Gerisch, G. (2002). Dynamics of the vacuolar $\mathrm{H}^{+}$-ATPase in the contractile vacuole complex and the endosomal pathway of Dictyostelium cells. J. Cell Sci. 115, 2893-2905.

Clarke, M., Maddera, L., Engel, U., and Gerisch, G. (2010). Retrieval of the vacuolar H-ATPase from phagosomes revealed by live cell imaging. PLoS ONE 5:e8585. doi: 10.1371/journal.pone.0008585

Cox, G. M., McDade, H. C., Chen, S. C., Tucker, S. C., Gottfredsson, M., Wright, L. C., et al. (2001). Extracellular phospholipase activity is a virulence factor for Cryptococcus neoformans. Mol. Microbiol. 39, 166-175. doi: 10.1046/j.1365-2958.2001.02236.x

Derengowski, L. da. S., Paes, H. C., Albuquerque, P., Tavares, A. H. F. P., Fernandes, L., Silva-Pereira, I., et al. (2013). The transcriptional response of Cryptococcus neoformans to ingestion by Acanthamoeba castellanii and macrophages provides insights into the evolutionary adaptation to the mammalian host. Eukaryot. Cell 12, 761-774. doi: 10.1128/EC.00073-13

Derivery, E., Sousa, C., Gautier, J. J., Lombard, B., Loew, D., and Gautreau, A. (2009). The Arp2/3 activator WASH controls the fission of endosomes through a large multiprotein complex. Dev. Cell 17, 712-723. doi: 10.1016/j.devcel.2009.09.010

Evans, R. J., Li, Z., Hughes, W. S., Djordjevic, J. T., Nielsen, K., and May, R. C. (2015). Cryptococcal phospholipase B1 is required for intracellular proliferation and control of titan cell morphology during macrophage infection. Infect. Immun. 83, 1296-1304. doi: 10.1128/IAI.03104-14

Feldmesser, M., Kress, Y., Novikoff, P., and Casadevall, A. (2000). Cryptococcus neoformans is a facultative intracellular pathogen in murine pulmonary infection. Infect. Immun. 68, 4225-4237. doi: 10.1128/IAI.68.7.4225-4237.2000

Fu, M. S., and Casadevall, A. (2018). Divalent metal cations potentiate the predatory capacity of amoeba for Cryptococcus neoformans. Appl. Environ. Microbiol. 84: e01717-17. doi: 10.1128/AEM.01717-17

Gilbert, A. S., Seoane, P. I., Sephton-Clark, P., Bojarczuk, A., Hotham, R., Giurisato, E., et al. (2017). Vomocytosis of live pathogens from macrophages is regulated by the atypical MAP kinase ERK5. Sci Adv 3:e1700898. doi: 10.1126/sciadv.1700898

Gomez, T. S., and Billadeau, D. D. (2009). A FAM21-containing WASH complex regulates retromer-dependent sorting. Dev. Cell 17, 699-711. doi: 10.1016/j.devcel.2009.09.009

Griffiths, R. E., Kupzig, S., Cogan, N., Mankelow, T. J., Betin, V. M. S., Trakarnsanga, K., et al. (2012). Maturing reticulocytes internalize plasma membrane in glycophorin A-containing vesicles that fuse with autophagosomes before exocytosis. Blood 119, 6296-6306. doi: 10.1182/blood-2011-09-376475

Johnston, S. A., and May, R. C. (2010). The human fungal pathogen Cryptococcus neoformans escapes macrophages by a phagosome emptying mechanism that is inhibited by Arp2/3 complex-mediated actin polymerisation. PLoS Pathog. 6:e1001041. doi: 10.1371/journal.ppat.1001041

Johnston, S. A., and May, R. C. (2013). Cryptococcus interactions with macrophages: evasion and manipulation of the phagosome by a fungal pathogen. Cell. Microbiol. 15, 403-411. doi: 10.1111/cmi.12067

Johnston, S. A., Voelz, K., and May, R. C. (2016). Cryptococcus neoformans thermotolerance to avian body temperature is sufficient for extracellular growth but not intracellular survival in macrophages. Sci. Rep. 6:20977. doi: 10.1038/srep20977

King, J. S., Gueho, A., Hagedorn, M., Gopaldass, N., Leuba, F., Soldati, T., et al. (2013). WASH is required for lysosomal recycling and efficient autophagic and phagocytic digestion. Mol. Biol. Cell 24, 2714-2726. doi: 10.1091/mbc.E13-02-0092

Le Roux, D., Le Bon, A., Dumas, A., Taleb, K., Sachse, M., Sikora, R., et al. (2012). Antigen stored in dendritic cells after macropinocytosis is released unprocessed from late endosomes to target B cells. Blood 119, 95-105. doi: 10.1182/blood-2011-02-336123

Ma, H., Croudace, J. E., Lammas, D. A., and May, R. C. (2006). Expulsion of live pathogenic yeast by macrophages. Curr. Biol. 16, 2156-2160. doi: 10.1016/j.cub.2006.09.032

Nosanchuk, J. D., and Casadevall, A. (2006). Impact of melanin on microbial virulence and clinical resistance to antimicrobial compounds. Antimicrob. Agents Chemother. 50, 3519-3528. doi: 10.1128/AAC.00545-06

Noverr, M. C., Cox, G. M., Perfect, J. R., and Huffnagle, G. B. (2003). Role of PLB1 in pulmonary inflammation and cryptococcal eicosanoid production. Infect. Immun. 71, 1538-1547. doi: 10.1128/IAI.71.3.15381547.2003

Peters, S., Reinthal, E., Blitgen-Heinecke, P., Bartz-Schmidt, K. U., and Schraermeyer, U. (2006). Inhibition of lysosomal degradation in retinal pigment epithelium cells induces exocytosis of phagocytic residual material at the basolateral plasma membrane. Ophthalmic Res. 38, 83-88. doi: $10.1159 / 000090268$

Rajasingham, R., Smith, R. M., Park, B. J., Jarvis, J. N., Govender, N. P., Chiller, T. M., et al. (2017). Global burden of disease of HIV-associated cryptococcal meningitis: an updated analysis. Lancet Infect. Dis. 17, 873-881. doi: 10.1016/S1473-3099(17)30243-8

Ravdin, J. I., Murphy, C. F., and Schlesinger, P. H. (1988). The cellular regulation of vesicle exocytosis by Entamoeba histolytica. J. Protozool. 35, 159-163.

Seaman, M. N., Gautreau, A., and Billadeau, D. D. (2013). Retromer-mediated endosomal protein sorting: all WASHed up! Trends Cell Biol. 23, 522-528. doi: 10.1016/j.tcb.2013.04.010

Smith, L. M., Dixon, E. F., and May, R. C. (2015). The fungal pathogen Cryptococcus neoformans manipulates macrophage phagosome maturation. Cell. Microbiol. 17, 702-713. doi: 10.1111/cmi.12394

Steenbergen, J. N., Nosanchuk, J. D., Malliaris, S. D., and Casadevall, A. (2003). Cryptococcus neoformans virulence is enhanced after growth in the genetically malleable host Dictyostelium discoideum. Infect. Immun. 71, 4862-4872. doi: 10.1128/IAI.71.9.4862-4872.2003

Steenbergen, J. N., Shuman, H. A., and Casadevall, A. (2001). Cryptococcus neoformans interactions with amoebae suggest an explanation for its virulence and intracellular pathogenic strategy in macrophages. Proc. Natl. Acad. Sci. U.S.A. 98, 15245-15250. doi: 10.1073/pnas.261418798

Steinman, R. M., Brodie, S. E., and Cohn, Z. A. (1976). Membrane flow during pinocytosis. A stereologic analysis. J. Cell Biol. 68, 665-687.

Stukes, S., Coelho, C., Rivera, J., Jedlicka, A. E., Hajjar, K. A., and Casadevall, A. (2016). The membrane phospholipid binding protein annexin A2 promotes phagocytosis and nonlytic exocytosis of Cryptococcus neoformans and impacts survival in fungal infection. J. Immunol. 197, 1252-1261. doi: 10.4049/jimmunol.1501855

Tenforde, M. W., Scriven, J. E., Harrison, T. S., and Jarvis, J. N. (2017). Immune correlates of HIV-associated cryptococcal meningitis. PLoS Pathog. 13:e1006207. doi: 10.1371/journal.ppat.1006207

Turner, C. T., Fuller, M., Hopwood, J. J., Meikle, P. J., and Brooks, D. A. (2016). Drug induced exocytosis of glycogen in Pompe disease. Biochem. Biophys. Res. Commun. 479, 721-727. doi: 10.1016/j.bbrc.2016.09.145

Tucker, S. C., and Casadevall, A. (2002). Replication of Cryptococcus neoformans in macrophages is accompanied by phagosomal permeabilization and accumulation of vesicles containing polysaccharide in the cytoplasm. Proc. Natl. Acad. Sci. U.S.A. 99, 3165-3170. doi: 10.1073/pnas.052702799

Voelz, K., Johnston, S. A., Rutherford, J. C., and May, R. C. (2010). Automated analysis of cryptococcal macrophage parasitism using GFP-tagged cryptococci. PLoS ONE 5:e15968. doi: 10.1371/journal.pone.0015968

Watkins, R. A., King, J. S., and Johnston, S. A. (2017). Nutritional requirements and their importance for virulence of pathogenic 
Cryptococcus species. Microorganisms. 5:E65. doi: 10.3390/microorganisms5 040065

Weisman, R. A., and Korn, E. D. (1967). Phagocytosis of latex beads by Acanthamoeba. I. Biochemical properties. Biochemistry 6, 485-497. doi: 10.1021/bi00854a017

Wiesner, D. L., Specht, C. A., Lee, C. K., Smith, K. D., Mukaremera, L., Lee, S. T., et al. (2015). Chitin recognition via chitotriosidase promotes pathologic type2 helper T cell responses to cryptococcal infection. PLoS Pathog. 11:e1004701. doi: 10.1371/journal.ppat.1004701

Zech, T., Calaminus, S. D., Caswell, P., Spence, H. J., Carnell, M., Insall, R. H., et al. (2011). The Arp2/3 activator WASH regulates alpha5beta1-integrin-mediated invasive migration. J. Cell Sci. 124(Pt 22), 3753-3759. doi: 10.1242/jcs.080986
Conflict of Interest Statement: The authors declare that the research was conducted in the absence of any commercial or financial relationships that could be construed as a potential conflict of interest.

Copyright (c) 2018 Watkins, Andrews, Wynn, Barisch, King and Johnston. This is an open-access article distributed under the terms of the Creative Commons Attribution License (CC BY). The use, distribution or reproduction in other forums is permitted, provided the original author(s) and the copyright owner are credited and that the original publication in this journal is cited, in accordance with accepted academic practice. No use, distribution or reproduction is permitted which does not comply with these terms. 